\title{
Nonlinear Optics of Nanostructures
}

\author{
Vladimir I. Gavrilenko, ${ }^{1}$ Tatiana V. Murzina, ${ }^{2}$ and Goro Mizutani ${ }^{3}$ \\ ${ }^{1}$ Department of Physics, Center for Materials Research, Norfolk State University, Norfolk, VA 23504, USA \\ ${ }^{2}$ Department of Physics, Moscow State University, Moscow 119991, Russia \\ ${ }^{3}$ Japan Advanced Institute of Science and Technology, School of Materials Science, 1-1 Asahidai, Ishikawa, Nomi 923-1292, Japan \\ Correspondence should be addressed to Vladimir I. Gavrilenko, vgavrilenko@nsu.edu
}

Received 31 October 2012; Accepted 31 October 2012

Copyright (C) 2012 Vladimir I. Gavrilenko et al. This is an open access article distributed under the Creative Commons Attribution License, which permits unrestricted use, distribution, and reproduction in any medium, provided the original work is properly cited.

The optical properties of nanostructures are fascinating and useful for a variety of applications. Nanotechnologies are poised to revolutionize medicine, manufacturing, energy production, and other fundamental features of everyday life in the 21st century. The unique nonlinear optical properties of nanoscale materials are size dependent; they do not naturally occur in larger bulk materials. The selected collection of papers included in this special issue demonstrates that nonlinear optics of the bulk contrasts markedly with the current state of knowledge about the optics of surfaces and interfaces in nanomaterials. It is challenging to understand optical properties of different complex materials (aggregated molecular and polymer systems, crystalline and noncrystalline solids) at the microscopic scale.

One of the major objectives of nanoscience is a detailed understanding of the physics and chemistry behind the interaction between objects (surfaces, particles, and individual molecules) at the nanoscale. Questions need to be answered regarding how nanoparticles can be stabilized, and in what media, how nanoparticles interact and influence each other and solid surfaces, and what proportions in a hybrid system make a critical difference. How can these characteristics be realistically predicted and extracted from nonlinear optics? The papers of this special issue address the following topics:

(i) experimental technique of nonlinear optics in nanomaterials;

(ii) theory of nonlinear optical response from surfaces and nanostructures;

(iii) modeling and simulation of nonlinear optical functions in nanomaterials; (iv) nonlinear optics of surfaces and interfaces; surface steps and imperfections; low-dimensional systems, quantum wells, and nanowires; nanoplasmonic structures and metamaterials; chiral nanostructures; nanostructured photonics crystals; micro- and nanocavities, ring cavities; excitons in nanoparticles; self-assembled monolayers; biophotonic crystals;

(v) electrical- and magnetic-field-induced nonlinear optics in surfaces and nanostructures;

(vi) nonlinear magnetooptics of nanostructures and magnetophotonic crystals;

(vii) Terra-Herz generation in nanoparticles;

(viii) ultrafast nonlinear spectroscopy of nanoparticles.

Second harmonic generation (SHG) data from $\mathrm{Si}(001) /$ $\mathrm{SiO}_{2}$ heterostructures and multiquantum well systems are reviewed by T. V. Murzina et al. The SHG response from these samples comes from the thin interface layers containing information about specifics of the microscopic structures on $\mathrm{Si}(001)-\mathrm{SiO}_{2}$ interface.

Transient atomic vibration of $\mathrm{Cu}(111)$ covered with alkali metals is studied using ultrafast time-domain SHG technique by K. Watanabe and Y. Matsumoto. They discovered that the initial phases of the oscillating component of SHG intensity after the excitation pulse reflects the origin of the relevant electronic excitation at the metal-metal interface.

A nonlinear optical microscope developed to produce three-dimensional images of second harmonic generation from polar surfaces is described by $\mathrm{H}$. Yokota et al. This approach represents activity in the development of next generation nonlinear optical diagnostics of solid surfaces and interfaces. 
The theoretical paper by Zh. Kudyshev et al. is focused on generalized analytical solution for nonlinear wave propagation in waveguide couplers. Quite a general case of the system is considered characterized by linear refractive indexes with opposite signs, nozero phase mismatch between the channels, and arbitrary nonlinear coefficients.

SHG generation from the Pt-nanowire arrays fabricated on $\mathrm{MgO}(110)$ templates is reviewed by Y. Ogata and G. Mizutani. A contribution of roughness has been discussed in a context of the nanowire array radiation sensitivity.

All contributions to this issue contain analysis of trends and suggestions towards a rapid development of the nonlinear optical diagnostics of nanostructures.

Vladimir I. Gavrilenko Tatiana V. Murzina Goro Mizutani 

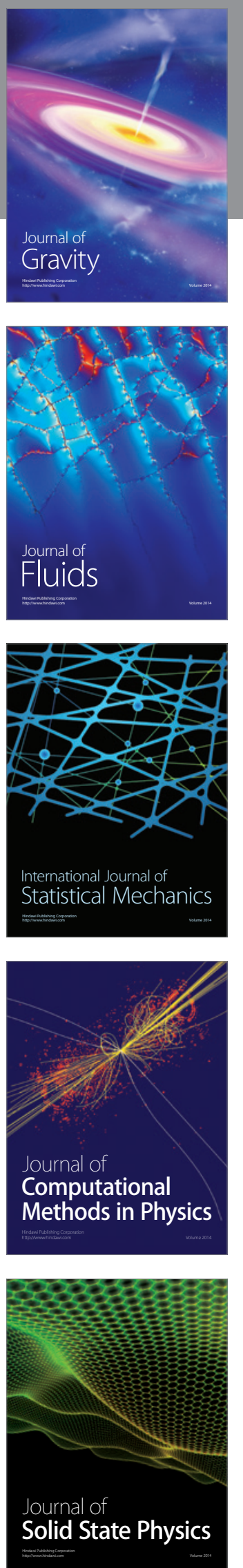

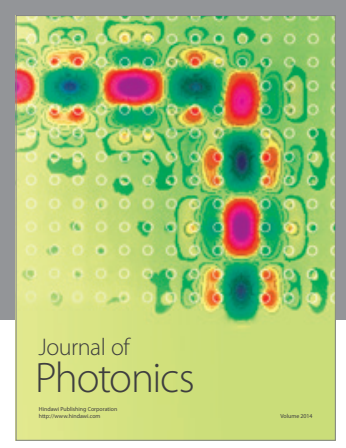

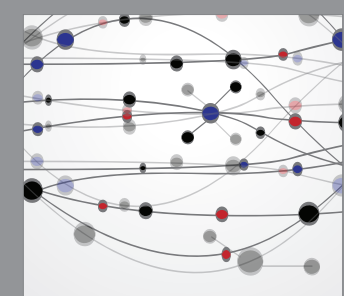

The Scientific World Journal
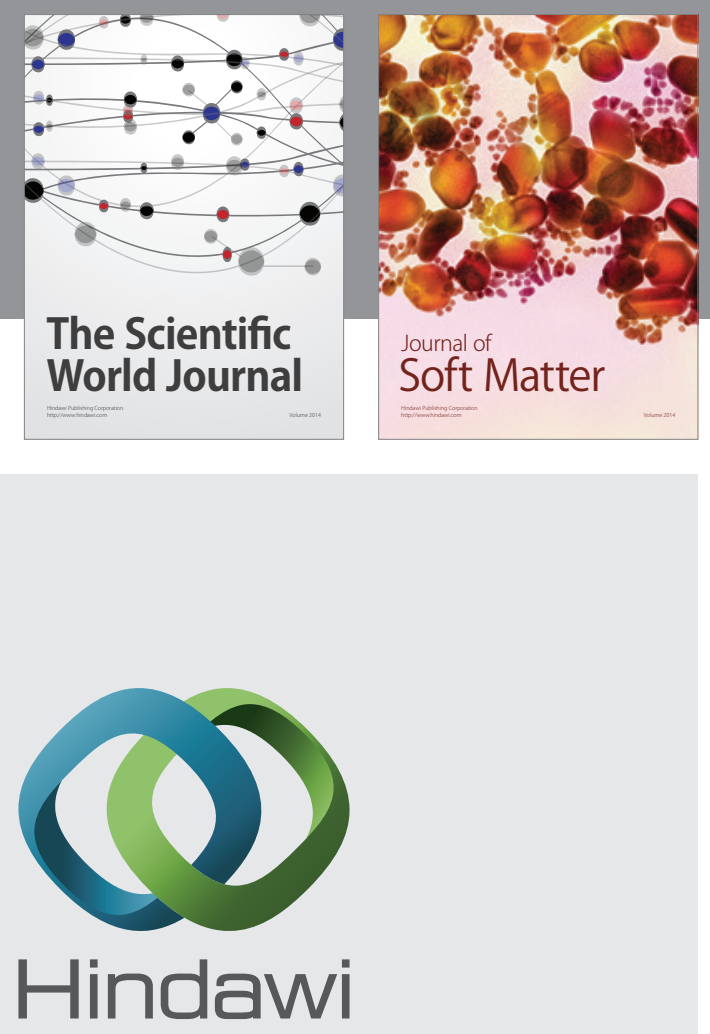

Submit your manuscripts at

http://www.hindawi.com
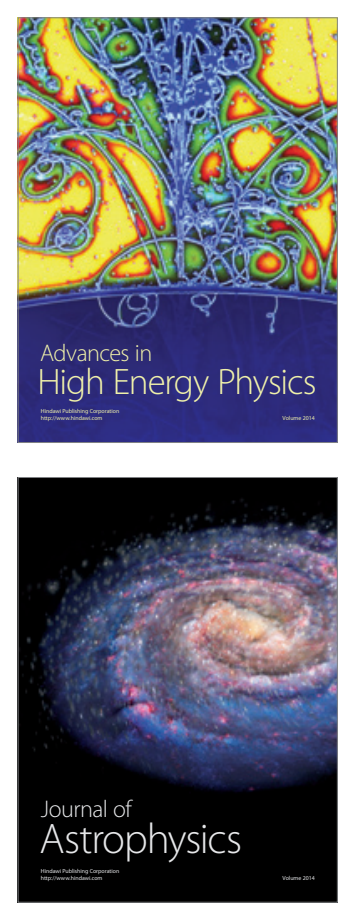
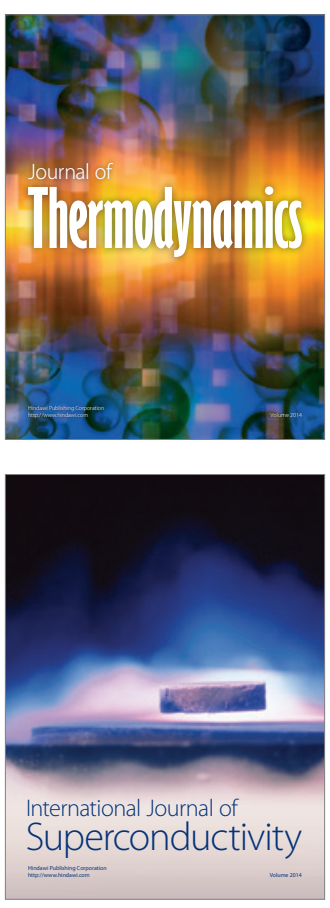
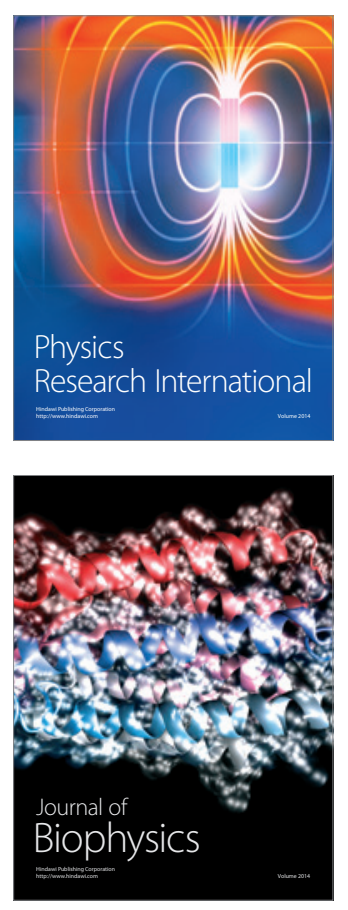
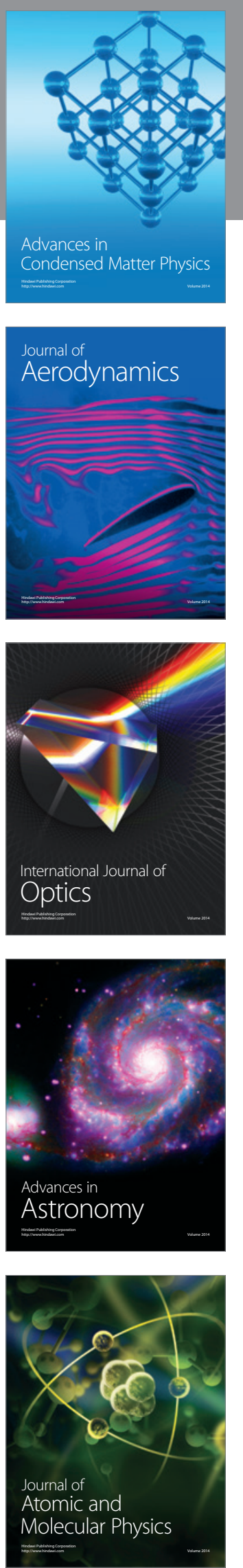